\title{
El Modelo Sintético de Comunicación de Niklas Luhmann
}

\author{
NIKLAS LUHMANN'S SyNTHETICAL MOdEL OF COMMUNiCATION
}

Mg. Sergio Pignuoli-Ocampo (spignuoli@conicet.gov.ar) Consejo Nacional de Investigaciones Científicas y Técnicas (Argentina) y Facultad de Ciencias Sociales, Universidad de Buenos Aires (Buenos Aires, Argentina)

\begin{abstract}
In this paper we review the critical reception given by Niklas Luhmann's General Theory of Social Systems to the classical Information Theory, as basis of its postulation of a General Sociological Theory entirely founded on the Theory of Communication. Our hypothesis suggests that the formulation of the synthetic model of communication re-formulates the classical model of Shannon, through a replacement of tele-communicative accents by sociological accents.
\end{abstract}

Keywords: communication, information, Luhmann, social systems theory, Shannon

\section{Resumen}

En este trabajo revisamos la recepción crítica dada por la teoría de sistemas sociales de Niklas Luhmann a la teoría de la información clásica, en el marco de su postulación de una teoría general de la sociología fundada en la teoría de la comunicación. La hipótesis que defendemos es que la formulación del modelo sintético de la comunicación reformula el modelo clásico de Shannon, mediante una sustitución de los acentos telecomunicativos por acentos sociológicos.

Palabras clave: comunicación, información, Luhmann, teoría de sistemas sociales, Shannon

\section{Introducción}

A la hora de fundamentar y direccionar la innovación en el terreno de la teoría sociológica, el sociólogo alemán Niklas Luhmann revalidó la tensión entre tradiciones sociológicas y campos interdisciplinarios (teoría de sistemas, teoría de la complejidad, teoría evolutiva, teoría de la emergencia) y desarrolló diversas estrategias para usufructuarla. Un rasgo distintivo de todas ellas fue remarcar las limitaciones que la sociología se auto-imponía al aislarse de los avances de otros campos científicos. Para remediarlas el autor reubicó la discusión interdisciplinaria en el corazón de sus innovaciones. En este trabajo analizamos la estrategia que empleó en 1984 para reformular el concepto sociológico de comunicación y para establecer un modelo sintético de esta, según el cual la comunicación emerge como síntesis de tres selecciones: Información / Emisión (Mitteilung) / Comprensión (Verstehen) (1).

A partir de los años noventa, tras ser eclipsado por las polémicas de los años ochenta sobre la autopoiesis y el antihumanismo, el concepto luhmanniano de comunicación comenzó a recibir la atención debida. Tempranamente Darío Rodríguez y Marcelo Arnold (1991:113) destacaron su importancia para la concreción del giro de la teoría general de sistemas a la teoría de sistemas sociales. Eva Knodt (1995: xxiv) puso al "giro comunicativo" en pie de igualdad con el "giro autopoiético". Rudolph Stichweh (2000:8) llevó la revaluación hasta su cima, al afirmar la centralidad de la elevación luhmanniana de la comunicación al rango de concepto fundamental de la disciplina. Desde entonces y hasta la actualidad este concepto no sólo conservó, sino que aumentó su vigencia, sobre todo si lo comparamos con otras perspectivas comunicativas, más renombradas en el campo a comienzos de los años ochenta (como la teoría de la acción comunicativa de Habermas o la hermenéutica del entendimiento de Gadamer). 
Desde nuestra óptica, enmarcados dentro de una línea de investigación interesada en profundizar y problematizar los vínculos entre doble contingencia y comunicación en la TGSS, consideramos que la pertinencia sociológica del concepto sintético de comunicación de Luhmann es indisociable de la amplia recepción y del uso sociológico dados por el autor a la teoría clásica de la información y a la pragmática interpersonal de la Escuela de Palo Alto (2). Su recepción crítica de estas últimas puso en dialogo los avances científicos producidos en esos campos con las limitaciones de las tradiciones sociológicas para tratar la comunicación (incluida la escuela accionalista). Por eso, a nuestro criterio, es necesario integrar ambos cuestionamientos y desarrollar un argumento que explicite la estrategia interdisciplinaria que lo soportó, para ello profundizamos el modelo sintético de comunicación planteado por Luhmann, en 1984, y lo situamos dentro de la tensión entre limitaciones disciplinarias, aperturas interdisciplinarias y alternativas teóricas. Nuestro objetivo es optimizar el diálogo con ambos cuestionamientos, ofreciendo un panorama más amplio y a la vez más preciso de la estrategia epistemológica llevada adelante por Luhmann, y, además, ampliar la visión restringida que simplifica el dialogo de la TGSS con la teoría de la información a la sola crítica de "la metáfora de la transmisión".

Nuestra hipótesis sugiere que Luhmann desarrolló una teoría sociológica de la comunicación a partir de modelos no-sociológicos de comunicación, tras criticar las tradiciones dominantes del campo (accionalismo, colectivismo y giro lingüístico) pero sin dejar de proponerles su teoría como una alternativa ante sus respectivas dificultades. El modelo sintético de 1984 supone una recepción crítica de la teoría clásica de la información por parte de la TGSS y constituye una reformulación, desde los intereses de la sociología, del modelo informacional de comunicación de Shannon (en adelante: MIC). Nuestra hipótesis rechaza que Luhmann haya establecido una sociología matemática de la comunicación, y niega a la vez que el uso del MIC se haya limitado a criticar la "metáfora de la transmisión". Sugerimos más bien que el uso del MIC por parte de la TGSS está regido por un procedimiento interdisciplinario, a saber, desarrollar un concepto sociológico de comunicación que cubra una vacancia que no podía satisfacerse desde las tradiciones del campo sociológico. Para ello reformuló el MIC, lo que exigió la eliminación y sustitución de los acentos telecomunicativos del MIC por acentos sociológicos. La TGSS realizó estas operaciones de eliminación y sustitución bajo el título de crítica de la "metáfora de la transmisión", aunque no alteró los materiales sino sólo los acentos, por lo que también sugerimos, como sub-hipótesis, que Luhmann dio un uso sociológico de los materiales del MIC y que esta selección alejó al modelo sintético de las tradiciones sociológicas, que tomaban habitualmente como materiales al lenguaje, al sujeto, a la consenso simbólico o a la comunidad.

La exposición que sigue a continuación seguirá este orden, revisaremos en primer lugar las discusiones que la TGSS sostuvo contra los modelos con que la sociología subsumió tradicionalmente a la comunicación y contra las propuestas de "giro lingüístico" de la disciplina (sección 2); tras ello estudiamos el modelo informacional clásico, la discusión cibernética sobre él (sección 3), y el uso sociológico dado por Luhmann a la cuestión (sección 4), allí reconstruimos la definición conceptual de comunicación (sección 5). Finalmente presentamos las conclusiones y su discusión a la luz del debate sobre la pertinencia sociológica del concepto de comunicación (sección 6).

\section{El contexto disciplinario del concepto sintético de comunicación de la TGSS}

La TGSS de Niklas Luhmann es el último proyecto teórico de la sociología con envergadura general, pretensión universalista y recepción global. Si bien la TGSS aspiró desde temprano a constituir la disciplina en una teoría de los sistemas sociales (Luhmann 2009a:143), su desarrollo, sin embargo, no fue homogéneo. Más allá de algunas continuidades puntuales, Luhmann modificó la morfología y los fundamentos de su proyecto en varias oportunidades. Podemos periodizar su evolución en tres momentos: el primero (1964-1984) se caracteriza por la asunción de las teorías de la acción y la teoría funcional cibernética de sistemas. El segundo (1984-1990) se caracteriza por la asunción de las teorías de la comunicación y de sistemas autorreferenciales autopoiéticos clausurados en la operación. El punto de inflexión es la publicación de Sistemas sociales, en el que apareció el modelo sintético (3). El tercero (1990-1997) se caracteriza por profundizar el vínculo epistemológico y metodológico con las teorías de la forma de Spencer Brown y de los observing systems de von Foerster. El punto de inflexión es la publicación de La ciencia de la sociedad (4).

El concepto de comunicación tuvo su propia evolución dentro de este proceso. Registró un gran número de variaciones durante el primer periodo. Si bien, según Stichweh (2000:7), en las producciones de Luhmann de ese 
periodo convivieron "aproblemáticamente" los conceptos acción, comunicación y sistema, las variaciones provinieron de la apertura interdisciplinaria del autor y su continua recepción crítica de conceptos de la teoría clásica de la información y de la pragmática interpersonal. La reformulación general de 1984 marcó la estabilización del concepto. En ese momento, Luhmann postuló el modelo sintético de la comunicación como operación de los sistemas sociales junto con las tesis de la emergencia y la autopoiesis comunicativas de dichos sistemas. Desde entonces, el concepto ya no sufrió mayores modificaciones.

La reformulación de 1984 convirtió al programa luhmanniano en el primer programa sociológico general fundado en la comunicación. La comunicación no fue concebida como tema de una sociología especial ni como una noción promisoria, sino como la categoría fundamental de la perspectiva teórica de la disciplina. La historia del concepto sociológico de comunicación es extensa, sin embargo, hasta el "giro lingüístico" de los años setenta, es una historia secundaria de la disciplina. Este relegamiento no se debió a un desinterés temático -la comunicación nunca fue ajena a la sociología-, sino más bien, a la asimetría de un prius lógico. Los intereses "generales" de la disciplina la subsumieron bajo categorías y problemas entendidos como "mayores" (la modernidad, la estructura social, la ideología, las clases, entre las más salientes). Así, hallamos dos modelos clásicos de subsunción sociológica de la comunicación. El primero de ellos es el modelo individualista, que tiende a reducirla a la formulación de enunciados por parte de un actor que utiliza el intercambio simbólico con fines instrumentales o intencionados. El segundo modelo es el holista, que la entiende como uno de los medios a través de los cuales se transmiten transparentemente determinaciones sociales desde un punto (o una parte) de una determinada unidad hacia otros puntos (o partes) de la misma (o de una otra) unidad, y coadyuvan así en la reproducción de determinadas entidades o estructuras supraindividuales, postuladas como "colectivas" o "societales"; en este modelo la comunicación también es entendida como un tipo de acción entre otros, pero ya no está sujeta al arbitrio egoísta de un actor, sino a las necesidades funcionales socio-reproductiva de tales entidades.

En el contexto de la nueva "crisis de la sociología" de los años setenta, los modelos de subsunción recibieron fuertes objeciones. Los aspectos más criticados fueron la desatención del estatuto sociológico del lenguaje y el retraso relativo de la disciplina respecto de la lingüística. Para remediar esta debilidad surgieron diversas tentativas de reformulación que pusieron el foco de las innovaciones en la modificación del concepto y del estatuto del lenguaje. La evidente metaindividualidad del lenguaje ofrecía un soporte incuestionablemente colectivo para delimitar los objetos, los problemas y las intervenciones de las ciencias sociales. La premisa básica es que el lenguaje es constitutivo de la realidad social, como fenómeno tiene una historicidad y una estructuración específicas, y, debido a su eficacia estructurante, organiza y clausura el sentido. De esto se desprendió una perspectiva sobre la comunicación, la que queda regida por los principios de la constitución y de la estructuración lingüística, y así es enfocada por el giro lingüístico como un conjunto de prácticas y circulaciones discursivas que organizan y reproducen la estructura social.

Luhmann participó de la discusión sociológica sobre el concepto de comunicación con anterioridad a 1984. Heintz (2004:21) señaló que las intervenciones del autor estaban informadas por la aspiración de generar una teoría sociológica general alternativa tanto al individualismo metodológico como al holismo colectivista; contra el primero alegó que lo social no puede ser explicado de manera suficiente por ninguna propiedad unilateral relativa a sujetos, actores o agentes individuales; contra el segundo, que lo social no es un a priori ni una instancia del mundo enajenada de la interacción entre al menos dos participantes. Luhmann dialogó, en cambio, con perspectivas afines a la "revuelta microsociológica" (un heterogéneo movimiento de "tercera vía" alternativo a las tradiciones mencionadas, aparecido hacia los años sesenta) con las que compartían un común interés por formular un concepto de lo social enfocado en la "interacción", a la que declaraba irreductible a la unilateralidad de una conciencia ni deducible de entidades "en sí" sociales (sociedad, estructuras sociales, etc.). En este sentido observamos que Luhmann rechazó en general los modelos de subsunción porque obstaculizaban la observación de la autonomía social (y sociológica) de la comunicación. En cuanto a cada modelo de subsunción en particular, Luhmann rechazó la premisa del modelo individualista porque consideró que el hacer comunicativo sólo es posible dentro de los sistemas sociales dónde el sentido social se organiza en la referencia recíproca de las acciones, y no en la enunciación unilateral de un actor (Luhmann 2009b: 186; 2009c: 12; 2009d: 104). En cuanto al modelo colectivista, Luhmann rechazó por simplista la premisa de que la comunicación funcione como un medio 
transparente de transferencia de significados, y le contrapuso la tesis de que la comunicación produce, "actualiza" sentido de un modo específico en y por el hacer entre los hombres, y dadas la no-identidad y la intransparencia recíproca entre ellos, la "transferencia" de sentido de uno hacia otro es imposible (Luhmann 1971:42) (5).

En cuanto al "giro lingüístico" de las ciencias sociales, Luhmann se mostró reacio a resolver problemas sociológicos con "soluciones" extrapoladas del ámbito lingüístico. A juicio del autor, dicho "giro" padecía tres problemas: 1) generaba equívocos en la definición del objeto, porque aun cuando el lenguaje sea una entidad incuestionablemente colectiva, no es un medio privativo de los sistemas sociales, pues también lo emplean los sistemas psíquicos, por ello no puede predicarse de él que sea un objeto exclusiva ni necesariamente social; 2) subsumía de modo inapropiado la comunicación al lenguaje, pues, al no ser capaz de organizarse ni de generar sus propios elementos, el lenguaje no constituye ningún sistema, y por ende es incapaz de clausurar el sentido de la comunicación (y de la conciencia). Para la TGSS el lenguaje sólo funciona socialmente cuando es empleado por el hacer comunicativo y sólo en carácter de medio de comunicación que probabiliza el entendimiento, y 3) imponía a la comunicación un telos de entendimiento. En abierta polémica con Habermas, Luhmann rechazó la idea de que el lenguaje sea un medio de intercambio simbólico regido por premisas de una racionalidad del entendimiento, ni mucho menos que su horizonte sea la integración social ni una intersubjetividad de acuerdos. Para Luhmann, el código comunicativo del medio lingüístico es "sí (aceptación) / no (rechazo)", esta estructura binaria garantiza la posibilidad de rechazo de cualquier comunicación. Por ende ponderar sólo uno de los valores ese código, en este caso la aceptación, conduciría a una visión incompleta (y restrictiva) del lenguaje, y, además, llevaría a predicar de modo inválido que el "no (rechazo)" sería menos propio de lo social que el "sí (aceptación)".

Este conjunto de críticas llevó a Luhmann a afirmar que el concepto de comunicación constituía una vacancia en la sociología, pues se carecía de una perspectiva específicamente disciplinaria sobre el problema. Para contrarrestar las limitaciones de las tradiciones disciplinarias, el autor apeló al dialogo interdisciplinario con dos tradiciones nosociológicas: la pragmática interpersonal de la Escuela de Palo Alto y la teoría clásica de la información.

Con la Escuela de Palo Alto, Luhmann mantuvo un dialogo complejo y amplio. Por una parte, se mostró tempranamente interesado por los conceptos pragmáticos de Paul Watzlawick y su equipo. De ellos retomó las nociones de "percepción recíproca" (con la cual sustituyó la pretensión de reciprocidad plena entre comunicantes por la percepción de la diferencia para que haya comunicación) y de "situación de imposible no-comunicación" (por la cual ponderó la situación por sobre la acción y enfatizó el peso de la situación de la doble contingencia para la selección de la comunicación). Además, por otra parte, Luhmann se inclinó por la distinción entre comunicación y metacomunicación de Jürgen Ruesch y Gregory Bateson. Estos autores pusieron de relieve, de modo pionero hacia comienzos de los años cincuenta, que la comunicación no es sólo un proceso informativo sino también un proceso de coordinación de acciones, y distinguieron la comunicación de una información (el mensaje) de las reglas sintácticas y semánticas así como de la conducta pragmática mediante las cuales es enunciado dicho mensaje. Luhmann reformuló la distinción básica, como señaló Stichweh (2000: 8), y distinguió la información (o mensaje) de la acción intencionada de un actor por la cual da a conocer dicha información. Con ello se contrapuso al modelo de subsunción individualista, pues criticaba la premisa de la unilateralidad de la acción no sólo porque es incapaz de explicar la unidad social de la comunicación, sino porque tampoco abarca la unidad de la acción de enunciación. Con esta recepción, Luhmann profundizó la matriz interaccional/mutualista del concepto sociológico de comunicación, en detrimento del reduccionismo accionalista, pues lo vinculó con la investigación pragmática de la relación específica entre producción de información y la necesidad de coordinación de acciones.

En tanto, de la teoría clásica de la información, Luhmann retomó el modelo CPM de Donald MacKay (por el cuál rechazó la tesis transmicionista y asumió que la comunicación es una selección que reduce complejidad apoyada en la diferencia entre sentido e información) (6), y, luego, el análisis conversacional de Gordon Pask (por el cual rechazó la tesis fenomenológica de la constitución del sentido y asumió la emergencia de las expectativas mutualísticas) (7). Con esta recepción Luhmann deslindó al concepto sociológico de comunicación de la ontología, pues lo definió como un acontecimiento contingente que sucede dentro del mundo, pues su especificidad es reducir complejidad mediante una selección de sentido compleja, para cuya constitución es necesario el concurso y la coordinación de al menos dos acciones en el marco de expectativas de acción (condición que la vuelve 
necesariamente social), y cuya unidad de la diferencia entre sentido e información la vuelve irreductible no sólo a la intención de los actores sino también a las entidades metaindividuales como la "sociedad" o el "lenguaje".

Al entrar en la década del ochenta, Luhmann ya había alcanzado una reformulación muy importante del concepto sociológico de comunicación. En ese momento, radicalizó el concepto elevándolo al rango de categoría fundamental. Uno de los pasos que condujeron a dicha elevación fue la postulación del modelo sintético de comunicación, el cual afirma que la comunicación es la síntesis de tres selecciones, para lo cual incorporó un nuevo dialogo interdisciplinario al concepto, esta vez con el modelo clásico de Shannon.

\section{Uso del modelo informacional clásico}

Dadas las deficiencias de los modelos sociológicos, la TGSS optó por desarrollar una teoría sociológica de la comunicación a partir de modelos no-sociológicos de comunicación. Esto justifica que la TGSS haya usado el modelo informacional de comunicación (MIC). Para seguir este movimiento revisaremos los postulados del MIC (3.1), luego, las críticas y las reformulaciones cibernéticas de él (3.2), y, finalmente, consideraremos las críticas y usos que Luhmann diera al MIC y a las críticas cibernéticas a la hora de definir sociológicamente la comunicación.

\subsection{El modelo informacional clásico (MIC)}

Lo que hoy conocemos como MIC fue originalmente denominado "teoría matemática de la comunicación". Ésta fue desarrollada por Claude Shannon y difundida por Warren Weaver a partir de finales de la década del cuarenta. Las investigaciones de Shannon estaban interesadas en desarrollar innovaciones de ingeniería telecomunicativa para los laboratorios de Bell System, filial de la empresa de telecomunicaciones American Telegraph \& Telephone (ATT). La novedad de aquellos planteos aún se estudia, dentro y fuera del campo de la telecomunicación.

El objetivo de Shannon era optimizar la correlación telecomunicativa entre una fuente de emisión, un canal de transmisión y una instancia de recepción. En ese entonces, la técnica estandarizada para aumentar la tasa de transmisión de información de un canal consistía en incrementar el poder, ampliando así el ancho de banda. Sin embargo, el procedimiento se mostraba ineficiente para transmitir señales a larga distancia debido a que el ensanchamiento de banda amplificaba también los ruidos de la transmisión. Ante este problema, la innovación de Shannon fue cambiar la perspectiva: propuso transmitir símbolos discretos en lugar de señales continuas. Para ello formuló un "sistema de comunicación general”, cuyo esquema básico está representado en la figura 1:

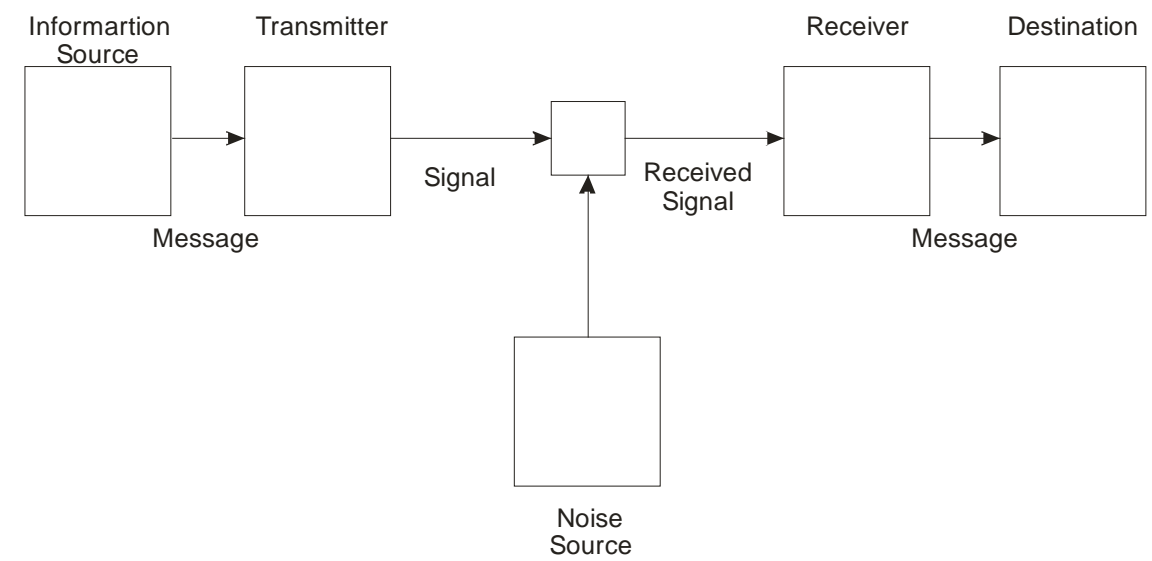

Fig. 1. Diagrama esquemático de un sistema general de comunicación (Shannon 1949:34)

Según el modelo de Shannon (1949:33), la comunicación es un sistema de transmisión discreta de mensajes que está organizado en función de una fuente, un mensaje, un canal y un receptor. Su funcionamiento es lineal y sigue estos pasos: 1) una fuente de información selecciona el mensaje deseado a partir de un conjunto de posibles 
mensajes o del repertorio de la fuente; 2) el transmisor transforma el mensaje en una señal que es enviada por el canal de transmisión/comunicación al receptor; 3) éste oficia de transmisor invertido (transforma la señal emitida en un mensaje) y 4) pasa este mensaje a su destinatario. Es característico de (2) que se agreguen a la transmisión ciertos elementos que no son deliberadamente proporcionados por la fuente de información; por lo cual se entienden como alteraciones en la señal denominadas 'ruido'.

Con estas premisas, Shannon comparó el rendimiento de distintos canales de transmisión (discreto/continuo) de acuerdo con el principio del ruido (canales con ruido y sin ruido [noiseless]) y realizó una importante serie de inferencias, entre las cuales se destacan las siguientes: 1) estableció la imposibilidad de eliminar la aparición de ruido en un canal de transmisión; 2) identificó la existencia de un umbral de inteligibilidad en función de la relación entre comprensión y ruido, y así postuló teóricamente el límite a partir del cual es tolerable el ruido del canal; 3 ) demostró que el uso de la redundancia de la señal optimiza el manejo del ruido del canal, independientemente del incremento del poder de señal; 4) determinó que para cualquier canal con ruido existen códigos. Solo es necesario sofisticar la codificación o encriptación de éstos de acuerdo con la cantidad de ruido del canal -aunque no demostró el método para hacerlo; 5) infirió que, si se cuenta con los códigos correctos, más ruido no significa mayor probabilidad de error, sino tan sólo mayor lentitud de transmisión.

Para nuestros objetivos resulta conveniente desarrollar dos conceptos contenidos en el catálogo: los conceptos de unidad selectiva de la información y de redundancia.

En cuanto a la unidad selectiva de la información, el MIC identificó su unidad de análisis en el contenido del mensaje, pero no en función de su significado sino de la relación entre información seleccionada, canal de transmisión e instrumento de codificación. En palabras de Rapoport (1984:73): “la cantidad de información de un mensaje está relacionada no con lo que se está diciendo sino con lo que podría decirse". Esa es la operación que distingue al MIC: examinar la diferencia selectiva que existe entre lo actual de un mensaje y lo posible de un código. Tal selección, y sólo ella, es información.

En cuanto al concepto de redundancia, cabe resaltar, en primer lugar, el sentido científico-técnico del movimiento de Shannon. Éste optó por resolver un problema técnico (la amplificación del ruido más allá de lo inteligible en las transmisiones a larga distancia) mediante la intervención de un factor no técnico (preconceptos sobre el contenido), posible de ser tecnificado (codificación de redundancias). Gracias a este movimiento, destructivo de cualquier diferenciación entre ciencias duras y blandas, el MIC resolvió incertidumbres técnicas con certidumbres no técnicas. En segundo lugar, la incorporación del concepto de redundancia dentro del proceso de transmisión, permitió a Shannon mediar la relación entre ruido e información, y así convertirla en una relación optimizable, con independencia de la variable técnica (cantidad de ruido del canal). En tercer lugar, el concepto de redundancia como conocimiento compartido de un código, tanto como verificación de la identidad de un mensaje transmitido, le ofreció al MIC el soporte suficiente para afirmar que la comunicación funciona como un sistema lineal de transmisión de información desde un determinado nodo hacia otro.

\subsection{Objeciones y reformulaciones cibernéticas}

Las investigaciones del campo de la cibernética hicieron del diálogo, la polémica y el uso del MIC una práctica constitutiva de ese campo. El momento fundacional de esta interfase entre campos nacientes es, sin dudas, la identificación de una analogía matemática entre la fórmula de Shannon para el cálculo de la cantidad de información $\left(\mathrm{H}=\log _{2} n\right)$ y la formula de Wiener para el cálculo de la entropía $\left(\mathrm{H}=-\sum p_{\mathrm{i}} \log p_{\mathrm{i}}\right)$, esta última, un fundamento de la cibernética, fue desarrollada en simultáneo con el trabajo de Shannon. Según Rapoport (1984:85) esta analogía matemática facilitó y potenció polémicas tanto como convergencias entre cibernética y MIC. Así, los primeros autores cibernéticos correlacionaron los conceptos de información y entropía. El propio Wiener entendió que la cantidad de información de un sistema es la medida de su grado de organización, y la cantidad de entropía es la medida de su grado de desorganización (Wiener 1948:76). La discusión cibernética llevó al MIC a otro nivel, haciéndolo intervenir en debates epistemológicos generales. Aquí revisaremos tres objeciones, que derivaron en importantes reformulaciones: la generalización del concepto de información (3.2.1), la organización circular (3.2.2) y la dimensión pragmática (3.2.3). 


\subsubsection{Generalización de los conceptos de información e intercambio}

Una de las objeciones típicas de la cibernética al MIC es la restricción arbitraria e injustificada que éste impone al concepto de información, al restringirlo a la ingeniería telecomunicativa. El trabajo cibernético a partir de la analogía matemática generalizó el concepto de información y lo volvió aplicable a todo tipo de sistemas. Una consecuencia fue que la idea de transmisión de información comenzó a ser entendida como subtipo de un fenómeno más general denominado intercambio de información. Éste constituye un mecanismo general de organización, cuya propiedad distintiva es la capacidad para regular la formación de patrones neguentrópicos (contrarios a la tendencia de la entropía). Esto se realiza o bien por la vía de la agregación de información y la consolidación de redundancias, o bien por la vía de la reacción ante eventos imprevistos o contrarios a la organización que requiere la producción más información.

\subsubsection{Organización circular}

El argumento por la generalización del concepto de información y la ponderación organizativa de la transmisión, derivó en nuevas objeciones. Una de ellas es la objeción contra la idea de que la organización de la transmisión de información sea lineal. Desde el punto de vista cibernético, el aporte del concepto de información es que permite observar que la transmisión de información genera organización circular, pues todo estado de una entidad organizada, e informacionalmente regulada, deriva de un intercambio previo de información. En ese sentido, aun cuando se conceda que la transmisión de mensajes a través de señales discretas es lineal, de ello no se desprende lógicamente que la organización sea lineal. El argumento cibernético afirma que ningún estado organizado es arbitrario, sino que es un estado determinado en función de una operación de intercambio de información anterior, esto indica la interacción entre información y organización. Uno de los usos más extendidos de este argumento lo constituye la perspectiva de que la organización es circular porque se retroalimenta informacionalmente, esto agrega al argumento básico la idea de que todo estado organizado supone tanto una retrospectiva como una prospectiva informacional. Wiener, Ashby y Maruyama son tres de los autores que mejor explotaron esa idea.

Norbert Wiener (1948:114) entendió que el mecanismo de regulación informacional es un mecanismo de reducción organizada del grado de entropía de cualquier entidad. La interacción entre organización e información está regulada por mecanismos de feedback. Esto significa que una entidad organizada produce información sobre la variación de sus propios estados. La función de esta información es disparar mecanismos de reversión de alteraciones y devolver la organización a un estado anterior a la alteración. La función de los feedback es ultraestabilizar la organización y su dinámica es de retroalimentación porque una organización ultraestable es capaz de autorregularse a través de interacciones específicas entre recursos e información propios.

En este punto, se vuelve relevante la revisión del MIC hecha por Ross Ashby (1957: 82-5; 242-3), otro de los padres fundadores de la cibernética. Ashby incorporó los conceptos de homeostasis y de ultraestabilidad a la transmisión de información, que él mismo había desarrollado a partir de su invención del homeostato. Según Ashby la información tiene una función homeostática porque implica la producción de estados propios a partir de información producida en función del entorno, gracias a ésta las entidades organizadas se orientan, regulan y estabilizan mediante una reorganización del intercambio de información con el ambiente resultante. Esto es cierto incluso en ambientes inestables o cambiantes, y es lo que se denomina ultraestabilidad. En este sentido, la regulación homeostática implica que la información es un auto-estado en función de un entorno.

Otro importante aporte cibernético provino de Magoroh Maruyama (1978:85). Éste llamó la atención sobre una deficiencia: el MIC no puede explicar el aumento de información. Para la epistemología "homogeneista" del MIC, la información, al igual que la entropía, tiende a decrecer. Esa es la razón por la que Shannon estudió y desarrolló principios capaces de subvertir esa tendencia, no para invertirla y volverla creciente, sino para contrariarla y mantenerla constante. Es un feedback negativo. El ideal de optimización de la transmisión de información así lo implica: la transmisión perfecta es aquella que mantiene constante la información del mensaje. Maruyama propuso atender la cibernética de los feedbacks positivos, aquéllos que, mediante la interacción y la desviación, tienden a incrementar la información y a generar estados organizados de un nuevo orden. Uno de los fenómenos predilectos de Maruyama es la aparición de una entidad novedosa a partir de la interacción entre patrones redundantes. La 
conclusión es que la redundancia no garantiza linealidad alguna, ni conduce por sí misma a la homeostasis, sino que es el tipo de interacción lo que conduce hacia uno u otro modo de organización circular, y la formación de ninguna redundancia es ajena a ello. La interacción entre organización e información no sólo es circular, sino además nolineal.

\subsubsection{La dimensión interactiva o pragmática}

El argumento por la organización circular derivó en nuevas objeciones. Una de ellas es que la lógica de la comunicación supone una organización circular de la interacción social, ausente de los modelos de optimización lineal de Shannon. El argumento pragmático básico es que la transmisión de información presupone interacción humana. Ésta constituye la comunicación y pone en juego determinado tipo de comportamientos, como el habla o la gestualidad, que cualquier miembro de un grupo puede codificar y decodificar como mensaje. A partir de estos comportamientos, la formación de patrones implica la formación de reglas, y la interacción regulada implica la formación de una organización social denominada red de comunicación. En la organización circular de la interacción, el receptor desempeña una función tan importante como el emisor.

Los psicólogos sociales fueron los primeros del campo de la investigación social en apreciar la fecundidad de los debates y las reformulaciones cibernéticas y en desarrollar modelos de análisis acordes con ellos. De acuerdo con la premisa de la transmisión y de la organización circular, Theodore Newcomb formuló el concepto de co-orientación. Co-orientación (u orientación simultánea) significa que dos seres humanos ( $\mathrm{A} ; \mathrm{B})$, al comunicarse entre sí, mantienen simultáneamente una doble orientación: 1) una orientación recíproca en cuanto comunicadores ( $A B$; $B A$ ), y 2) una orientación hacia un objeto (X) de la comunicación (AX; BX). Gracias a la inclusión de (2) -congruente con el concepto de transmisión-, Newcomb afirmó que todo acto de comunicación es una transmisión de información. Sobre ello desarrolló su famoso modelo ABX.

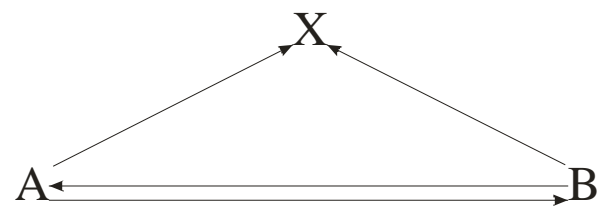

Fig. 2. Modelo ABX (Newcomb 1984:110)

Éste asume que la función de la comunicación es organizar la transmisión de información en un contexto de interacción, donde la co-orientación genera una interdependencia específica entre el conjunto de orientaciones $(A B ; B A ; A X ; B X)$ y conforma, así, un sistema circular, contrariamente a lo pensado por Shannon.

La versión más ambiciosa del argumento de la interacción humana pertenece, sin duda, a la "Escuela de Palo Alto", encabezada entonces por Bateson y Watzlawick. Su programa contó con un marco general, fases interdisciplinarias y definiciones epistemológicas. El marco diferenció el objeto comunicación en tres (información, significado, conducta) y distinguió tres perspectivas (sintaxis, semántica y pragmática) y tres unidades de análisis (redundancias lingüística, semántica e interactiva). Con arreglo a ello, el análisis de la conducta se diferencia del MIC sobre la base de la diferenciación del objeto, aunque aquél siga incluido dentro del marco de referencia. Mattelart y Mattelart (1997:48) subrayaron que la perspectiva de la Escuela de Palo Alto se distinguió por tres hipótesis fundamentales: 1) la comunicación se organiza como un proceso de relación e interacción, porque en ella prevalecen las relaciones sobre los elementos; 2) todo comportamiento humano tiene un valor comunicativo, por ello las relaciones se corresponden e implican mutuamente; 3) la sucesión de mensajes sigue una lógica (con un sentido horizontal y otro vertical) y conforma un sistema interpersonal. Nos permitimos agregar dos hipótesis fundamentales más: 4) la información produce una diferencia que es la condición para que se produzca otra diferencia y así las conductas se retroalimenten; 5) la comunicación estabiliza al sistema interpersonal porque es el vehículo del intercambio de información.

\section{Crítica y reformulación luhmannianas}

Luhmann enfocó la comunicación y la crítica del MIC desde una perspectiva emergentista. El MIC es el punto cero de la propuesta luhmanniana. Los materiales del modelo sintético de Luhmann no difieren de los empleados por 
aquél (8). En el MIC hay emisión, recepción e mensaje. En el modelo sintético hay síntesis de tres selecciones: la selección de Mitteilung (variación sobre el concepto de emisión), la selección de Verstehen (variación sobre el concepto de recepción) y la selección de información (variación sobre el concepto de mensaje). Para establecer el fundamento sociológico de su concepto de comunicación, la TGSS renunció a conceptos tradicionales como acción, cultura, lenguaje, consenso o comunidad, y sólo consideró necesario corregir la telecomunicación del MIC y acentuar sociológicamente los materiales y su unidad social.

La doble contingencia y las reformulaciones cibernéticas jugaron un papel preponderante en la corrección. Por una parte, las condiciones de la doble contingencia impiden que la comunicación pueda organizarse como "diálogo interior", el alter ego es exactamente eso: un alter ego, y el ego no puede ser un alter ego de sí mismo. Por otra parte, la síntesis no emerge como "transmisión", sino como unidad social de coordinación doble-contingente de selecciones. La TGSS se apoyó frecuentemente en las críticas y reformulaciones del MIC hechas desde la cibernética. Ésta también jugó un papel en la corrección sociológica del MIC hecha por Luhmann. A continuación desarrollamos las principales objeciones y reformulaciones que desembocaron en la definición de comunicación del modelo sintético de comunicación de la TGSS:

1) Paradoja de la incomunicabilidad. Luhmann objetó al MIC su completa omisión de la paradoja de la incomunicabilidad. Una consecuencia sociológica de primer orden del concepto de doble contingencia es la afirmación de que no es posible una relación inmediata entre conciencias. La TGSS prolongó esta afirmación por medios comunicativos, y concluyó que en virtud de esa imposibilidad es también imposible la comunicación entre conciencias. La paradoja surge porque gracias a esta incomunicabilidad interconciente hay comunicación; sólo que ésta ya no puede ser pensada como relación inmediata ni como transmisión de información entre conciencias, sino como emergente de aquella paradoja.

2) Diferencia entre información y sentido. Luhmann se interesó por la idea del MIC, proseguida por la cibernética, de que toda información supone selección entre posibilidades. Dicha idea resultaba congruente con el concepto general de sentido de la TGSS, ya que toda información adopta la forma de un estado actual sólo desde el punto de vista de una multiplicidad de estados posibles. Luhmann subordinó así el concepto de información al de sentido. La producción de información es un tipo de acontecimiento de sentido, cuya propiedad distintiva es la producir una novedad. Esta supone la producción de una diferencia y la alteración del estado de un sistema; sin embargo no todo procesamiento de sentido supone un acontecimiento de información. Todo procesamiento de sentido que no implique novedad, implica producción de sentido sin información.

3) La producción de información no supone intercambio, sino autorreferencia. A diferencia del MIC tanto como de la cibernética, Luhmann asume que los sistemas autorreferenciales están incapacitados para importar información. Necesariamente es producida ésta por las operaciones de un sistema. Tampoco hay homeostasis informacional entre sistema y ambiente, ya que un sistema autorreferencial está incapacitado para regularse por transfer intersistémico de información. Para la TGSS, una vez asumida la autorreferencia, información significa alteración del contexto interno de autodeterminación y, por tanto, necesariamente, auto-información. En consecuencia, la comunicación nunca implica transferencia, sino producción de información dentro de procesamientos de sentido.

4) Irrelevancia del requisito de identidad. Luhmann objetó al MIC que la unidad de la comunicación dependa exclusivamente de que se verifique la identidad de la información del mensaje transmitido. El soporte teórico ofrecido por la doble contingencia permite que la divergencia entre codificación y decodificación sea entendida como un presupuesto de toda comunicación, sin que ello obste para que haya una relación social. Así, la TGSS afirma que la unidad de la comunicación no surge de la identidad de información, sino de la relación social que necesariamente presupone.

5) Impertinencia de la ontología de la unilateralidad. Luhmann objetó el compromiso ontológico del MIC con la emisión. Para corregir ese sesgo unilateral, la TGSS señaló que, desde el punto de vista sociológico, la recepción es mucho más importante que la emisión. Ello radica en que, a diferencia de la emisión, si se verifica que existe una selección de recepción, se infiere la emergencia de una relación social, porque se presupone una emisión. La inferencia inversa no es válida, ni sociológicamente relevante. Así, la TGSS postula que la selección de Verstehen cierra la unidad social y sociológica de la comunicación. 
6) Crisis del modelo lineal. Luhmann se sumó a las objeciones contra el sentido lineal que el MIC impone a la comunicación. Para la TGSS, en primer lugar y de acuerdo con la DC y la cibernética de la interacción, la información no porta una dirección lineal, que va desde un emisor hasta un receptor, sino que existen distintos procesamientos simultáneos de sentido, cuya dirección es como mínimo de retroalimentación circular. En segundo lugar, Luhmann declaró falsa la premisa que restringe la selección de información a la emisión. La decodificación no es pasiva, sino que produce información, y la reintroduce en la unidad de la comunicación. En consecuencia, la información de la comunicación crece, al modo de Maruyama, y es más que la selección emitida emitida, como se ve en el siguiente esquema, que presentamos en un trabajo anterior (Pignuoli-Ocampo y Zitello 2011:937).

$$
\begin{gathered}
\text { (alter-emisor) [Emisión / Información } \left.n_{e}\left(I_{e}\right)\right] \\
\text { (ego-receptor) [Comprensión / Información } \left.{ }_{c}\left(I_{c}\right)\right] \\
{\left[\text { Emisión } / I_{e} / \text { Comprensión } / I_{c}\right] \rightarrow I_{e} / I_{c}=I_{s} \rightarrow[\text { Emisión / I } / \text { Comprensión] }}
\end{gathered}
$$

7) Síntesis vs. Dualismo. Luhmann objetó el dualismo con que el MIC vincula a las dos únicas selecciones (emisión, recepción) que identifica en la comunicación. Dados (3) y (6), la TGSS afirma que la comunicación consta de tres selecciones: la información también supone una selección propiamente comunicativa, no es un objeto ni un dato del mundo que esté ahí, dispuesto para que se comunique sobre él (como es el caso de la variable $X$ del modelo $A B X$ ). El dualismo telecomunicativo del MIC (que nos devolvería al modelo de subsunción individualista) no puede sino ser rechazado. La comunicación no tiene dos momentos individuales aislados y coordinados por una referencia objetual exterior, sino que emerge ya sintetizada, con una unidad social propia. Sus tres selecciones, incluida la información, son co-creadas dentro del proceso de la comunicación. Por tanto, el concepto de síntesis es el que mejor define la coordinación de las tres selecciones.

8) Omisión de la diferencia entre recepción y aceptación/rechazo. Luhmann objetó por injustificada la omisión del MIC de la distinción entre recepción y aceptación. Esta omisión acarrea dos dificultades, por un lado, la equiparación impide observar la improbabilidad de la aceptación y, por otro lado, renuncia a teorizar una cuarta distinción comunicativa: aceptar o rechazar el sentido de una comunicación con otra comunicación.

9) Omisión de la recursividad. Dada (8), Luhmann señaló que el MIC no puede ver cómo la comunicación se organiza circularmente, dado que una comunicación sólo puede ser aceptada o rechazada mediante otra comunicación que se enlaza a la anterior. De esta manera, la comunicación organiza procesos y estructuras autoreferidas en el nivel de los elementos a lo largo del tiempo. Esta red de comunicaciones recursivamente enlazada es el soporte fundamental del concepto de autopoiesis de los sistemas sociales.

10) Impertinencia del requisito de sincronía entre Emisión y Comprensión. Luhmann criticó al MIC su acotada perspectiva temporal, ya que al exigirle sincronía a la transmisión, volvió el concepto de comunicación inservible para analizar procesos comunicativos vueltos temporalmente más complejos, gracias a la mediación de la tecnología comunicativa (por caso: la escritura, la imprenta, la radio y teledifusión de ondas, la transmisión satelital y la codificación digital). El concepto de comunicación de la TGSS explota la posibilidad de que exista un desacoplamiento temporal y espacialmente entre recepción y emisión.

11) Tecnologicismo de la improbabilidad comunicativa. Luhmann objetó al MIC la visión tecnologicista de las improbabilidades de la comunicación. En contraposición afirmó que la improbabilidad técnica de la comunicación (el alcance, la propagación) no es la única. Cuando menos hay otras dos: la improbabilidad del entendimiento y la improbabilidad del éxito o aceptación (consecución). Un concepto sociológico de comunicación requiere romper con este tecnologicismo, sólo así podrá estudiarse la función comunicativa del lenguaje como medio de comunicación que probabiliza el entendimiento, y la evolución de los medios de comunicación simbólicamente generalizados como medios que probabilizan el éxito.

12) Asimetría. La linealidad de la transmisión postulada por el MIC presupone la simetría, pero no la asimetría de la comunicación. La comunicación, según Luhmann, posee la capacidad de autosimplificarse como acción y distribuir posiciones sociales de un modo asimétrico de acuerdo con cada una de las selecciones sintetizadas, así la 
comunicación se asimetriza y deviene acción, aunque toda posición es reversible. Esta autosimplificación representa una "fórmula de compromiso" para incluir a la acción dentro de la TGSS, bajo la condición de estar subordinada a la dinámica de la comunicación.

13) Las improbabilidades crecientes resultantes de la redundancia. La comunicación genera expectativas sobre la comunicación, de manera tal que la diferencia entre tema (expectativas temáticas) y aportes (contribuciones sobre un tema) coordina la recursividad del proceso comunicativo a partir de las propias expectativas comunicativas. La función de estas redundancias comunicativas no es disminuir la velocidad de transmisión, sino formar estructura y condicionar la probabilidad de la recursividad. Desde un punto de vista sociológico, al contrario del MIC, el crecimiento de redundancias es correlativo al aumento de la improbabilidad de la comunicación, pues aumenta la posibilidad de decepción.

Las críticas y reformulaciones tan variadas y agudas que propone Luhmann tienen algunos denominadores comunes. En primer lugar, hay un desplazamiento de los acentos telecomunicativos que el MIC puso sobre los materiales y un esfuerzo por generar una perspectiva que resalte tanto los componentes como la dimensión sociales de aquellos. En segundo lugar, las apoyaturas cibernética y sistémica apuntalaron a la doble contingencia a la hora de situar debidamente los acentos sociológicos. En tercer y último lugar, hay un punto de convergencia de todos los esfuerzos: orientar toda crítica y encaminar toda reformulación hacia la innovadora concepción sociológica de que la comunicación, emerge como una unidad social.

\section{Definición sociológica del concepto de comunicación}

La comunicación es definida como síntesis de tres selecciones: Información / Emisión / Comprensión. Se trata de una operación compleja, cuya unidad emergente se ordena del siguiente modo: una selección de "Información" (el tema de la comunicación, aquello sobre lo que se quiere expresar) es inicialmente procesada por un determinado emisor (alter) quien selecciona un determinado hacer en el mundo ("Emisión") -que puede ser una gesticulación, una locución oral, puesta por escrito, telecomunicativamente difundida o codificada simbólicamente- para que sea observado por un determinado receptor (ego) hacia quien tal "actuación" es dirigida. Sin embargo, dado que la comunicación es mutualista, no puede reducirse a la unilateralidad de una emisión, pues requiere que ego "actúe la comprensión" ("Comprensión") y distinga entre ese hacer del emisor en el mundo dirigido hacia él ("Emisión") y su decodificación de la "Información". Hay comunicación si, y sólo si, ego logra "actuar la comprensión". Leemos en Luhmann (1998:148): "Si se entiende la comunicación como síntesis de tres selecciones, como unidad de información, emisión y comprensión, entonces la comunicación se realiza cuando y hasta donde se genera comprensión. Todo lo demás sucede 'fuera' de la unidad elemental y la presupone".

Al cerrarse sintéticamente la unidad operativa de una comunicación, sólo podrá ser aceptada o rechazada mediante el enlace de una nueva comunicación que refiera a la comunicación anterior. La recursividad de estos enlaces entre comunicaciones genera un límite de sentido que posibilitará la diferenciación del sistema social.

La comunicación tiene dos propiedades operativas significativas: la propiedad reflexiva y la propiedad de autosimplificación. La propiedad reflexiva implica que puede tematizarse a sí misma. La propiedad de autosimplificación, que puede describirse a sí misma. El esquema que emplea para ello es el esquema de descripción de la acción.

La comunicación se organiza básicamente mediante la diferencia entre temas y aportes. La selección temática organiza las expectativas sobre la recursividad de comunicaciones que se indiquen a sí mismas como aportes referidos a dichas expectativas.

La comunicación tiene tres improbabilidades: 1) el entendimiento, 2) la propagación y 3) el éxito o consecución. La primera concierne a los problemas de codificación (primera y tercera selección), la segunda a los problemas de alcance (segunda selección) y la tercera a los problemas de enlace (cuarta selección). Las improbabilidades de la comunicación sólo pueden ser "desimprobabilizadas" por la comunicación, así se desarrollaron medios comunicativos referidos a cada improbabilidad. La improbabilidad del entendimiento es "desimprobabilizada" por el medio de comunicación del lenguaje, la improbabilidad del alcance por las tecnologías de propagación, y la improbabilidad del éxito o consecución por los medios de comunicación simbólicamente generalizados. 


\section{Conclusiones y discusión: pertinencia y alcance disciplinarios del concepto de comunicación}

La reformulación del modelo sintético de la comunicación indica una transformación de la posición de la TGSS al respecto de la relación entre acción y comunicación. Esta transformación va de la mano con las innovaciones en materia conceptual (la comunicación es entendida como unidad operativa de los sistemas sociales y como unidad de análisis de la sociología), en materia teórica (la comunicación destradicionaliza a la disciplina), y en materia interdisciplinaria (la comunicación dispone interfases con campos tradicionalmente desatendidos).

\section{La comunicación es el objeto unificado de la sociología}

Luhmann diagnosticó que el factor que ponía en crisis a la disciplina era la falta de una teoría general. La proliferación de "unidades de análisis" en el campo aumentó la presión sobre la identificación de un objeto sociológico unificado, dado que sin esto la pretensión de universalidad de cualquier teoría general que se postulara se derrumbaría. El modelo sintético de comunicación tuvo la función de satisfacer ese requisito. Para la TGSS, el modelo sintético indica inequívocamente que la comunicación es una operación que emerge necesariamente como unidad social, como "unidad mínima negable" (Luhmann 1998:153).

Dadas las propiedades operativas de toda comunicación, la TGSS reclamó que sea reconocido su carácter universal (define sociológicamente cualquier fenómeno social, sin exclusión de segmento social alguno) tanto como su carácter general (define sociológicamente qué es social y qué no). La comunicación tiene además propiedades evolutivas que indican que no es sólo una unidad de objeto sincrónico, sino que establece las premisas para el estudio de la evolución y la diferenciación de la comunicación, es decir, de lo social. No hay fenómeno "sociológico" que no presuponga la evolución de la comunicación. Para ello especifica las condiciones que hacen improbable lo social y estipula las condiciones para el desarrollo de medios con arreglo a ello. En virtud de ello, la TGSS consagró a la comunicación como unidad del objeto de la disciplina.

\section{La disciplina comunicativa critica masivamente a las tradiciones y a las innovaciones sociológicas}

El modelo sintético de comunicación representa en simultáneo una alternativa frente a gran conjunto de tradiciones teóricas de la sociología. Por un lado, radicaliza las tensiones y los problemas teóricos y conceptuales de buena parte de las tradiciones teóricas de la sociología, mientras les ofrece salidas a sus obstáculos epistemológicos, todas basadas en la opción por el emergentismo comunicativo. Por otro lado, ostenta la contraposición entre los giros comunicativo (al cual postula y defiende) y lingüístico (al que critica y desprecia). Este gesto, que entre otras cosas significa un distanciamiento de las sociologías "posmodernistas", muestra la correlación entre la reivindicación luhmanniana de la irreductibilidad del objeto y la pertinencia de la perspectiva sociológica. La TGSS correlaciona la solidez del modelo comunicativo con una recuperación de la solidez de la investigación sociológica dentro del sistema científico. En este sentido, ante las críticas de que pierde de vista la especificidad del objeto de la sociología y diluye la perspectiva sociológica construida por las tradiciones de la disciplina, la TGSS contrapone la afirmación programática de que el giro comunicativo refuerza las pretensiones científicas de la sociología, dándole un objeto unificado y una perspectiva general y altamente tecnificada. La TGSS no pone a la sociología ante el peligro de su desdiferenciación dentro del giro lingüístico, sino ante el riesgo de una destradicionalización de la disciplina y la promesa de una teoría general.

\section{Procedimiento interdisciplinario: agotar, reformular y retornar}

El uso del MIC como punto de partida para el desarrollo del modelo sintético de comunicación ofrece un ejemplo paradigmático de aquello que la TGSS entiende como uso disciplinario de circuitos interdisciplinarios para la formación de problemas, definiciones y marcos categoriales. Luhmann no encontró fundamentos tradicionales para tratar un problema eminentemente disciplinario. Desplegó una estrategia para explicitar esta grave falta y exponerla en sus fundamentos y luego buscó fundamentos sociológicos en campos e investigaciones concretas tradicionalmente "no sociológicas" como es el caso del MIC, la cibernética y la pragmática interpersonal. La demostración de la pertinencia del punto de vista sociológico para debatir en estos otros campos tuvo una doble función: por un lado, demostrar hacia la sociología que aquel "campo tradicionalmente no sociológico" no le era ajeno ni mucho menos irrelevante, y, por otro lado, revalorar la contribución de la sociológica en ámbitos interdisciplinarios. Gracias a esto, la TGSS reformuló sociológicamente el MIC y estableció el modelo sintético de la 
comunicación, y defendió su relevancia sociológica para establecer la unidad del objeto. Esta relevancia indicó el camino de retorno a la disciplina para reintroducir la solución. El resultado del procedimiento interdisciplinario fue postular una alternativa paradigmática, mantener sensibilizadas las interfases e interpelar al conjunto de las tradiciones del campo.

\section{Notas}

(1) A raíz de la dificultad suscitada por la traducción al español de las voces Mitteilung (traducido como participación, acto de comunicar, acción comunicativa) y Verstehen (traducido como comprensión, acto de entender) optamos por volcar Mitteilung como "Emisión”, al solo efecto de subrayar la dimensión comunicativa del término, en detrimento de las otras dimensiones de la voz. En tanto que para el término Verstehen nos ceñimos así a la larga tradición traductológica que lo ha volcado como "Comprensión". Más allá de esta decisión operativa, consideramos que hasta el momento, la mejor fórmula para volcar estos términos al castellano fue ofrecida por el equipo de traducción de La sociedad de la sociedad, liderado por el Dr. Javier Torres Nafarrate. El término Mitteilung fue volcado como "el-dar-la-información-a-conocer" y Verstehen como "el-entenderla". Cabe agregar que en las traducciones inglesas se ha generado una controversia similar, Mitteilung se ha traducido como utterance, act of utterance, imputation, y Verstehen como understanding, act of understand, comprehensive.

(2) Entre los resultados de nuestra línea de investigación podemos mencionar el análisis de fuentes de la doble contingencia en relación con las principales tradiciones disciplinarias del orden social (Pignuoli-Ocampo 2013), la evolución de la construcción del objeto sociológico en Luhmann y el impacto de la ponderación de la comunicación en su obra (Pignuoli-Ocampo 2012a), la optimización del manejo sociológico del problema de la alteridad a partir del concepto sintético de comunicación, en detrimento del concepto de dispositivo empleado por los principales referentes sudamericanos de los estudios poscoloniales (Pignuoli-Ocampo 2012b), así como un análisis crítico del concepto de comunicación de Luhmann (Pignuoli-Ocampo y Zitello 2011).

(3) Los primeros indicios de ese programa se observan ya en 1981. En el Prefacio al tercer volumen de Ilustración sociológica, Luhmann afirmó que el elemento de los sistemas sociales no era la acción ni un tipo especial de ellas como así tampoco un estilo de organización entre acciones (como había asumido desde los inicios de su carrera hasta ese momento), sino la comunicación, y sobre cuya base era posible desarrollar una perspectiva comunicativa sobre la acción. Sin embargo, la formulación del modelo sintético no hará su aparición sino hasta Sistemas sociales. Por lo tanto, entre 1981 y 1984 hay un subperíodo de transición en el que se suceden o conviven distintas formulaciones sobre la comunicación.

(4) Esta periodización está bajo estudio y la polémica la ha enriquecido y matizado. Kieserling (2002:171) advirtió acerca de la inconveniencia de hacer un corte muy tajante en 1984 en nombre de la introducción de la autopoiesis, pues según él los componentes sistémicos de la TGSS estaban terminados a comienzos de los setenta. En esa línea, Almaraz (1997) detectó continuidades entre los conceptos de sistema autorreferente y de sistema autopoiético y por ende entre las pretendidamente distantes primera y segunda fases; desde nuestra perspectiva, dicha continuidad es irrebatible y constituye un aporte relevante para el análisis de la evolución del pensamiento sistémico de Luhmann, sin embargo no consideramos pertinente difuminar la distinción entre ambas fases, puesto que en ese caso estaríamos omitiendo una discontinuidad mayor, a saber, el comienzo del primado sociológico del concepto de comunicación y la subordinación definitiva del concepto de acción.

(5) El uso de la noción de "transmisión” guarda un aspecto, aún poco estudiado, relativo a la aparición del término Übertragung en los primeros escritos de Luhmann. En algunos de estos (2009a: 159-163), el autor emplea el término para caracterizar la comunicación. Este uso atañe a sus primeras formulaciones de la teoría de los medios simbólicamente generalizados, a los que caracteriza, sin distinción alguna, como "medios de transmisión" o como "medios de comunicación". En 1971 se observa una separación entre medios de comunicación y funciones de transmisión, y en 1974 (2009e) la teoría de los MCSG predica de estos únicamente funciones de transmisión.

(6) Para vincular sentido y comunicación, Luhmann apeló al modelo CPM (Conditional-Probability-Matrix) de Donald Mackay (1969), pionero de la teoría británica de la información. Mackay sostuvo tempranamente que la teoría de sistemas no sólo requería de un concepto de información, sino también de un concepto de sentido. Sobre 
esa base desarrolló un modelo del sentido y de la información, que postula que el "sentido" es una función selectiva que para actualizarse supone una matriz de estados posibles. Dicha actualización selectiva de sentido es, a su vez, condición de posibilidad para la actualización de "información", que consiste de un "efecto sorpresa", un efecto de divergencia de las expectativas de sentido, que altera el estado de un sistema. Luhmann asumió tempranamente los principios de Mackay, a quién cita desde al menos 1969 para desarrollar un modelo no-lineal de procesamiento de sentido e información capaz de poner de relieve la autonomía social de la comunicación. Para Luhmann la comunicación no "transmite" sentido, sino que lo "actualiza" sobre la base de la diferencia entre sentido e información. Según sus palabras, la comunicación implica una "socialización de la sorpresa" (1971:43), por lo tanto no "transporta" sentido entre puntos distintos, sino que lo selecciona de una matriz de posibilidades (no es un mecanismo input-output), tampoco "transmite" información, sino que la actualiza como efecto específico sorpresivo que supone un determinado estado de expectativas y de alteraciones.

(7) Entrados los años ochenta, Luhmann abandonó la noción de constitución intersubjetiva del sentido, y retomó el concepto de conversation de Gordon Pask, el que empleó para afirmar que el sentido de la situación social supone una organización mutualística de expectativas, decisión que lo distanció de su primera asunción de la tradición fenomenológica para tratar esta cuestión.

(8) Cabe señalar que la reformulación del MIC de Roman Jakobson, una de las más importantes y difundidas, no fue tematizada por Luhmann. Ello explica por qué no discutió los factores "contexto" y "contacto" (este último una ampliación de Jakobson del concepto de Shannon de "canal", al que incorporó la dimensión de la "conexión psicológica" entre los hablantes). Al respecto merece una mención el análisis de Stahëli (2000:100) de la subsunción del factor "contexto" bajo la noción de selección y la inclusión limitada dentro del modelo sintético que ello propició, por lo cual Luhmann sustituyó la continuidad entre contexto de referencia y comunicación, con una síntesis de tres selecciones cada una con un horizonte. En cuanto al concepto de "contacto" debemos decir que Luhmann desarrolló en sus teorías de la doble contingencia y de la interpenetración una perspectiva sociológica mucho más compleja que la "conexión psicológica" entre hablantes, la que basó en la unidad social fundada en la alteridad y la diferencia. Finalmente, prologando algunas ideas de Rasch, puede hipotetizarse que Luhmann optó por reformular el MIC de Shannon, y no el de Jakobson, por la atención que el primero le deparó a los ruidos y a la unidad del proceso.

\section{Bibliografía}

Almaraz, J. 1997. Niklas Luhmann: la teoría de sistemas sociales antes de la autopoiesis. Revista Anthropos 173-174: 62-77.

Ashby, W. R. 1957. An introduction to cybernetics. London: Chapman \& Hall Ltd.

Heintz, B. 2004. Emergenz und Reduktion: Neue Perspektiven auf das Mikro-Makro-Problem. Kölner Zeitschrift für Soziologie und Sozialpsychologie 56(1): 1-31.

Kieserling, A. 2002. Rezension von Andreas Göbel. Kölner Zeitschrift für Soziologie und Sozialpsychologie 54(1): 171-173.

Knodt, E. M. 1995. Foreword. En: N. Luhmann. Social Systems. California: SUP, pp. ix-xxxvi.

Luhmann, N. 1971. Sinn als soziologische Grundbegriffe. En: N. Luhmann y J. Habermas. Theorie der Gesellschaft oder Sozialtechnologie? Frankfurt a.M.: Suhrkamp, pp. 25-100.

Luhmann, N. 1998 [1984] Sistemas sociales: lineamientos para una teoría general. México: Anthropos.

Luhmann, N. 2009a [1967] Soziologie als Theorie sozialer Systeme. Soziologische Aufklärung Vol. 1. Wiesbaden: VS Verlag, pp. 143-173.

Luhmann, N. 2009b [1969] Gesellschaft. Soziologische Aufklärung Vol. 1. Wiesbaden: VS Verlag, pp. 173-193.

Luhmann, N. 2009c [1975] Interaktion, Organisation, Gesellschaft. Soziologische Aufklärung Vol. 2. Wiesbaden: VS Verlag, pp. 9-24. 
Luhmann, N. 2009d [1973] Selbst-Thematisierung des Gesellschaftssystems. Soziologische Aufklärung Vol. 2. Wiesbaden: VS Verlag, pp. 89-127.

MacKay, D. 1969. Information, mechanism and meaning. Cambridge: MIT Press.

Maruyama, M. 1978. Heterogenistics and morphogenetics: toward a new concept of the scientific. Theory and Society 5(1): 75-96.

Mattelart, A. y Mattelart, M. 1997. Historia de las teorías de la comunicación. Barcelona: Paidos.

Newcomb, J. B. 1984. Por qué es necesario definir la comunicación. En: A. Smith (Comp.) Comunicación y cultura. Vol. 1. La teoría de la comunicación humana, Buenos Aires: Nueva Visión, pp. 91-110.

Pignuoli-Ocampo, S. 2013. Doble contingencia y orden social desde la teoría de Niklas Luhmann. Sociológica 78: 740 .

Pignuoli-Ocampo, S. 2012a. Los modelos de irreductibilidad social en Niklas Luhmann. Revista Española de Sociología 17: 27-48.

Pignuoli-Ocampo, S. 2012b. La noción de alteridad dentro de la pugna entre los marcos teóricos del dispositivo y de la comunicación. Nómadas: Revista Crítica de Ciencias Sociales y Jurídicas, Vol. Especial América Latina: 37-54.

Pignuoli-Ocampo, S. y Zitello, M. 2011. Tensiones y quiebres teóricos del concepto de comunicación de Luhmann. Estudios sociológicos 87: 925-947.

Rapoport, A. 1984. ¿Qué es la información? En: A. Smith (Comp.) Comunicación y cultura. Vol. 1. La teoría de la comunicación humana. Buenos Aires: Nueva Visión, pp. 71-90.

Rodríguez, D. y Arnold, M. 1991. Sociedad y teoría de sistemas. Santiago de Chile: Universitaria.

Shannon, C. 1949. The mathematical theory of communication. En: C. Shannon y W. Weaver. The mathematical theory of communication. Illinois: The University of Illinois Press, pp. 29-115.

Stäheli, U. 2000. Sinnzusammenbrüche. Göttingen: Velbrück Wissenschaft.

Stichweh, R. 2000. Systems theory as an alternative to action theory? The rise of 'communication' as a theoretical option. Acta Sociológica 43(1): 5-13.

Wiener, N. 1948. Cybernetics: or control and communication in the animal and the machine. New York: John Wiley \& Sons.

Recibido el 30 Nov 2012

Aceptado el 5 Feb 2013 